\title{
Highly Sensitive Porous PDMS-Based Capacitive Pressure Sensors Fabricated on Fabric Platform for Wearable Applications
}

\author{
Supporting Information
}

\section{Simin Masihił*, Masoud Panahił, Dinesh Maddipatla, Anthony J. Hanson, Arnesh K. Bose, Sajjad Hajian, Valliammai Palaniappan, Binu B. Narakathu, Bradley J. Bazuin and Massood Z. Atashbar*}

Department of Electrical and Computer Engineering, Western Michigan University, Kalamazoo, MI, USA

Stretchable TPU Film:

(a)

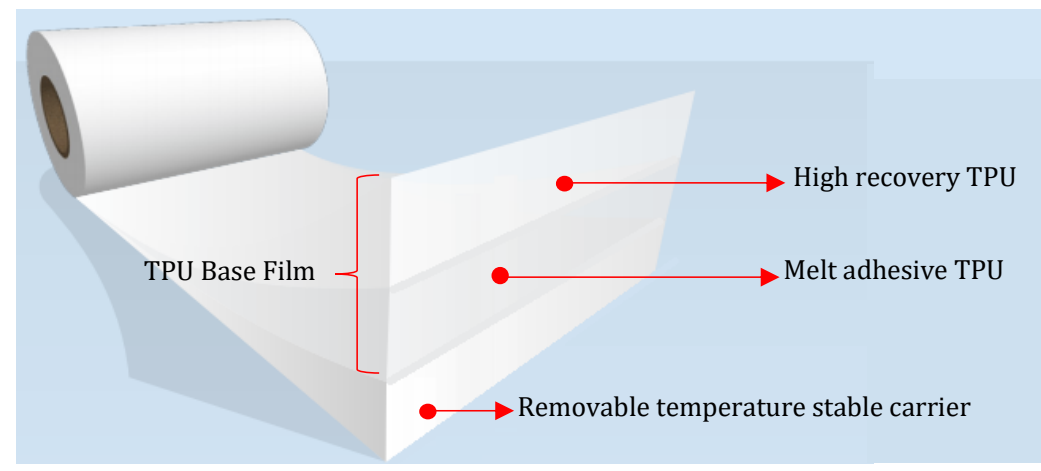

(b)

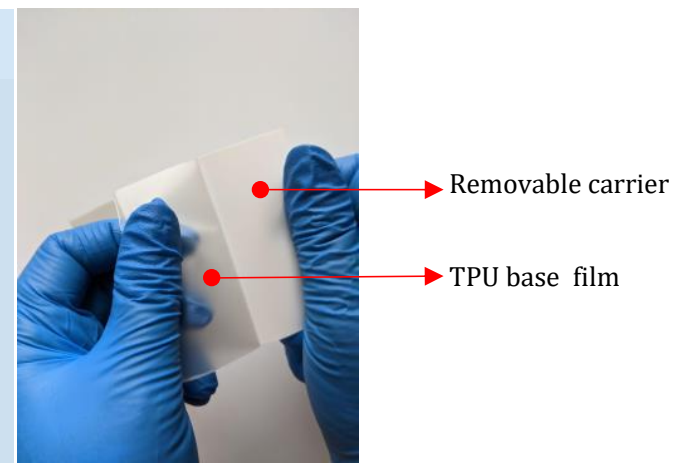

Figure S1: Stretchable bilayer thermoplastic polyurethane (TPU) film: (a) layers' illustration [https://www.ccieurolam.com/images/stories/pdf/PVAM_datasheets/Intexar-TE-11C.pdf] and (b) photograph of TPU film showing the base film and carrier.

\section{Reason for Choosing the Fabric and TPU for Electrode Fabrication:}

Wearability and breathability along with comfort are considered as one of the primary factors for players in sports applications. In addition, both flexibility and stretchablity are also important to be compatible with different head sizes and shapes specifically for this fit-cap application. Even though the TPU is stretchable enough for wearable applications, it does not provide the other aforementioned factors such as breathability and comfort unlike the Jennifer S/914 fabric (one of the finest sports cloths possessing superior stretch and recovery with advanced moisture management and comfort providing a better interface between sensor and sports wearables). However, the fabric is not compatible for direct deposition of metallic ink using printing process unlike TPU. Thus, the fabric and TPU were bonded together using heat lamination process (only in the sensing locations) to avail the best features of both, during the fabrication of the electrodes. 
(a)

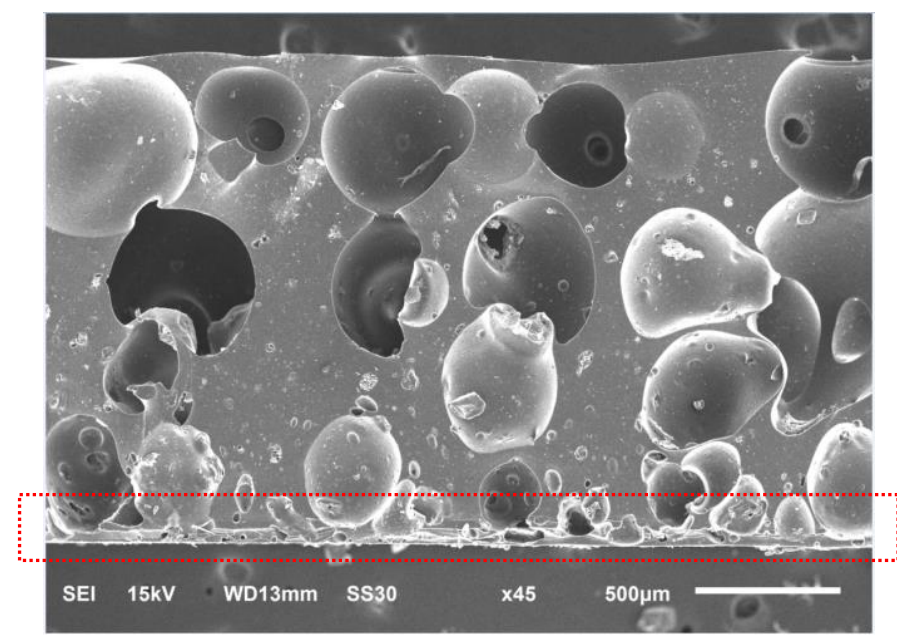

(b)

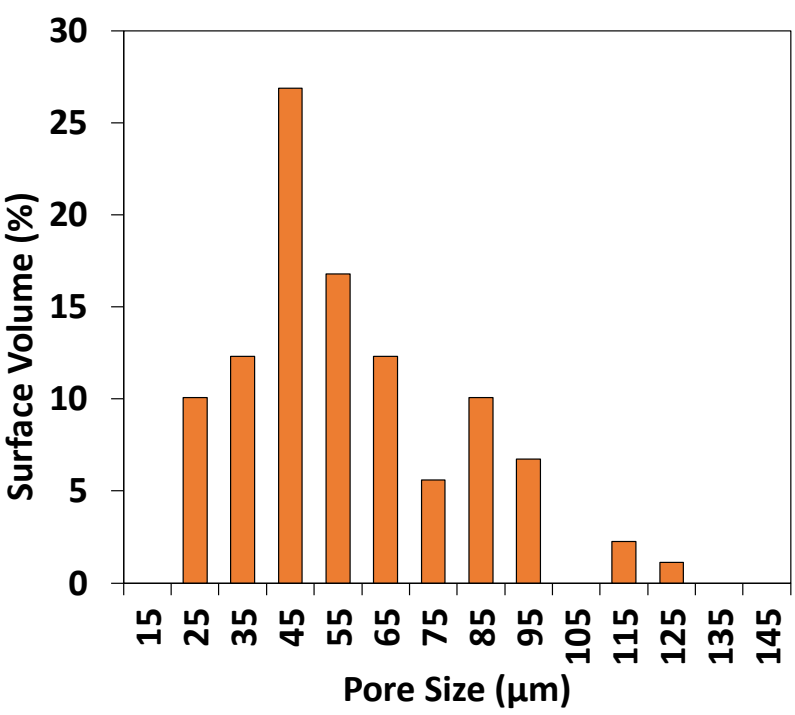

Figure S2. (a) SEM cross-sectional view of the porous dielectric layer D2 depicting the pores in the thickness of the layer including bottom pores and (b) pore distribution on the bottom side of D2.

Effect of Atmospheric Pressure Variations:

(a)

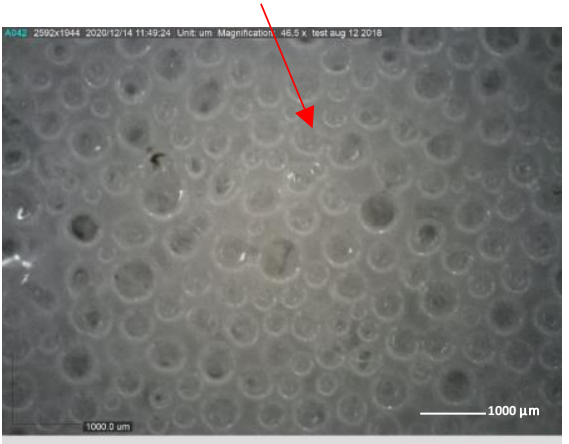

Thickness of layer $\sim 1680 \mu \mathrm{m}$ (b)

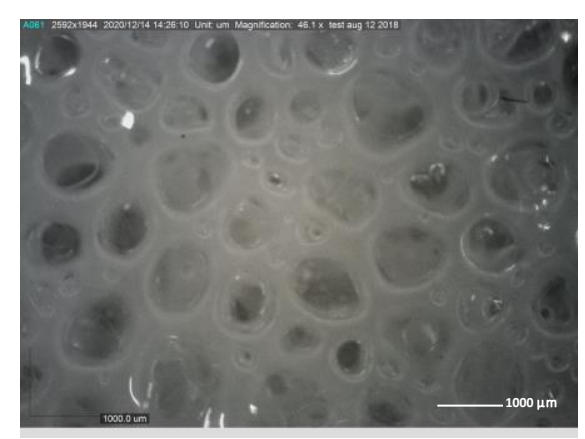

Thickness of layer $\sim 4170 \mu \mathrm{m}$ (c) Burst Gas Pocket (Hole)

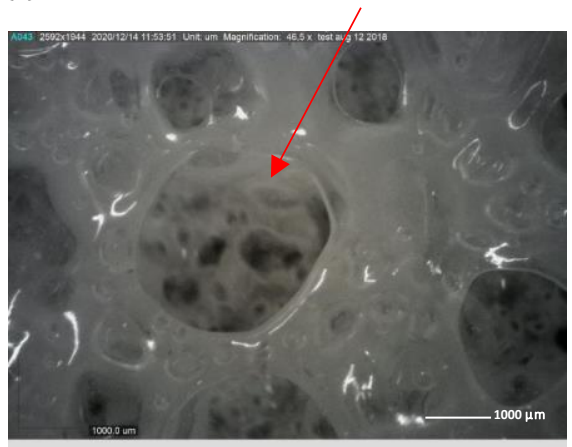

Thickness of layer $\sim 3748 \mu \mathrm{m}$

Figure S3. Microscopic images of the fabricated porous dielectric layer D2 cured under different pressures of: (a) regular atmospheric pressure ( $\sim 30 \mathrm{inHg}$ ), (b) 25\% reduced pressure (22.5 inHg) and (c) 50\% reduced pressure (15 inHg). 
Durometer Hardness, Tensile, and Compressive Properties of the Fabricated Layers:

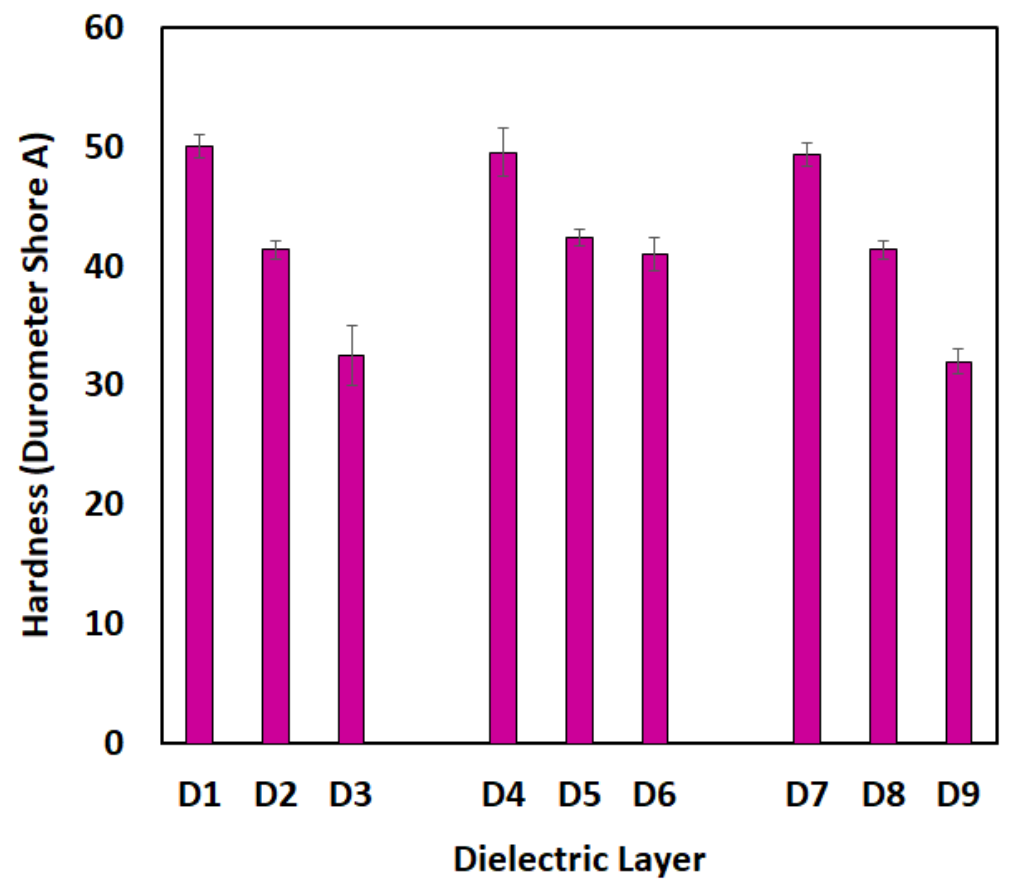

Figure S4. Hardness (durometer Shore A) for the for porous dialectic layers D1, D2, ...D9.

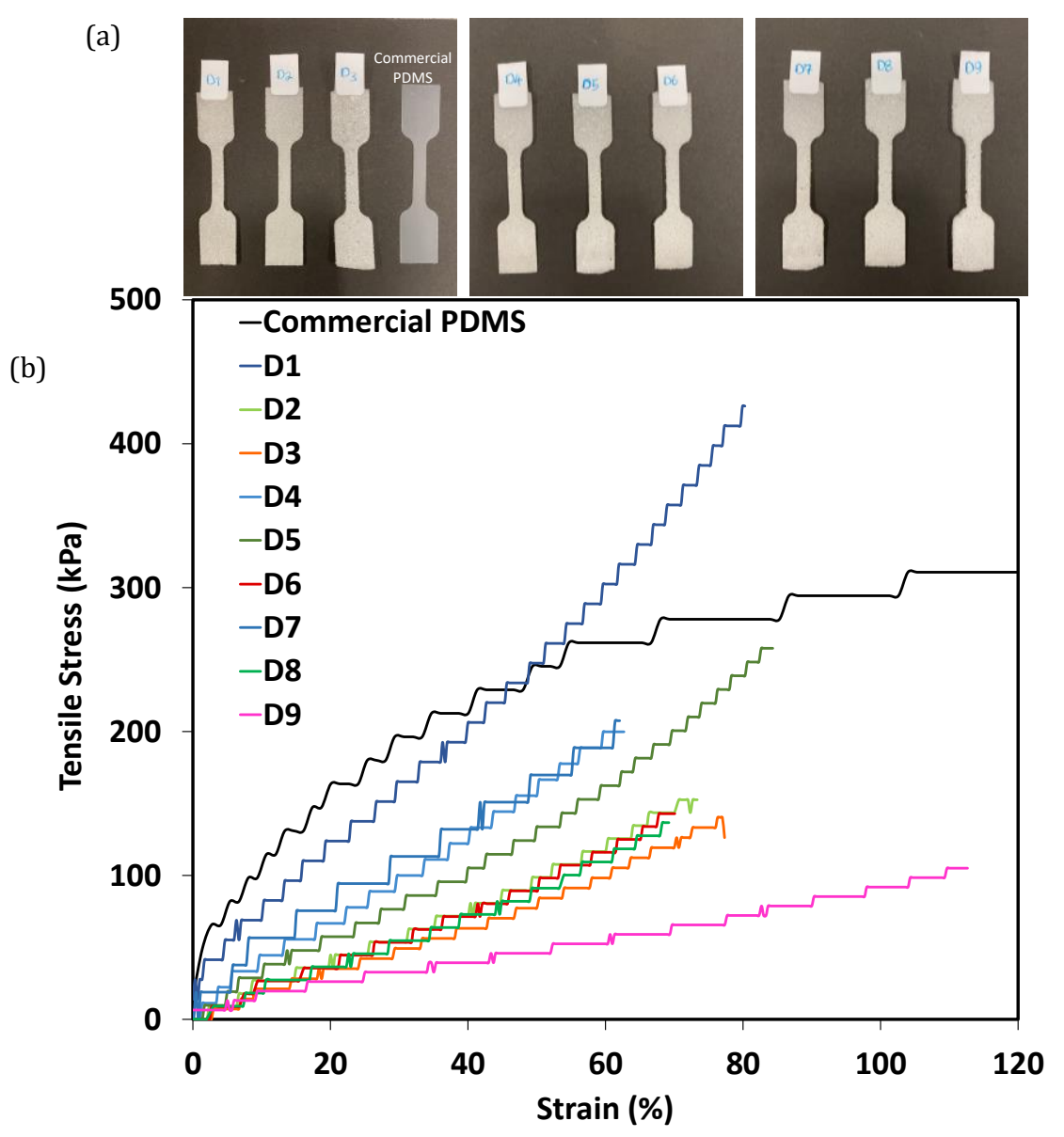


Figure S5.: Stress/strain curves showing the tensile deformation of porous dialectic layers D1, D2, ...D9.

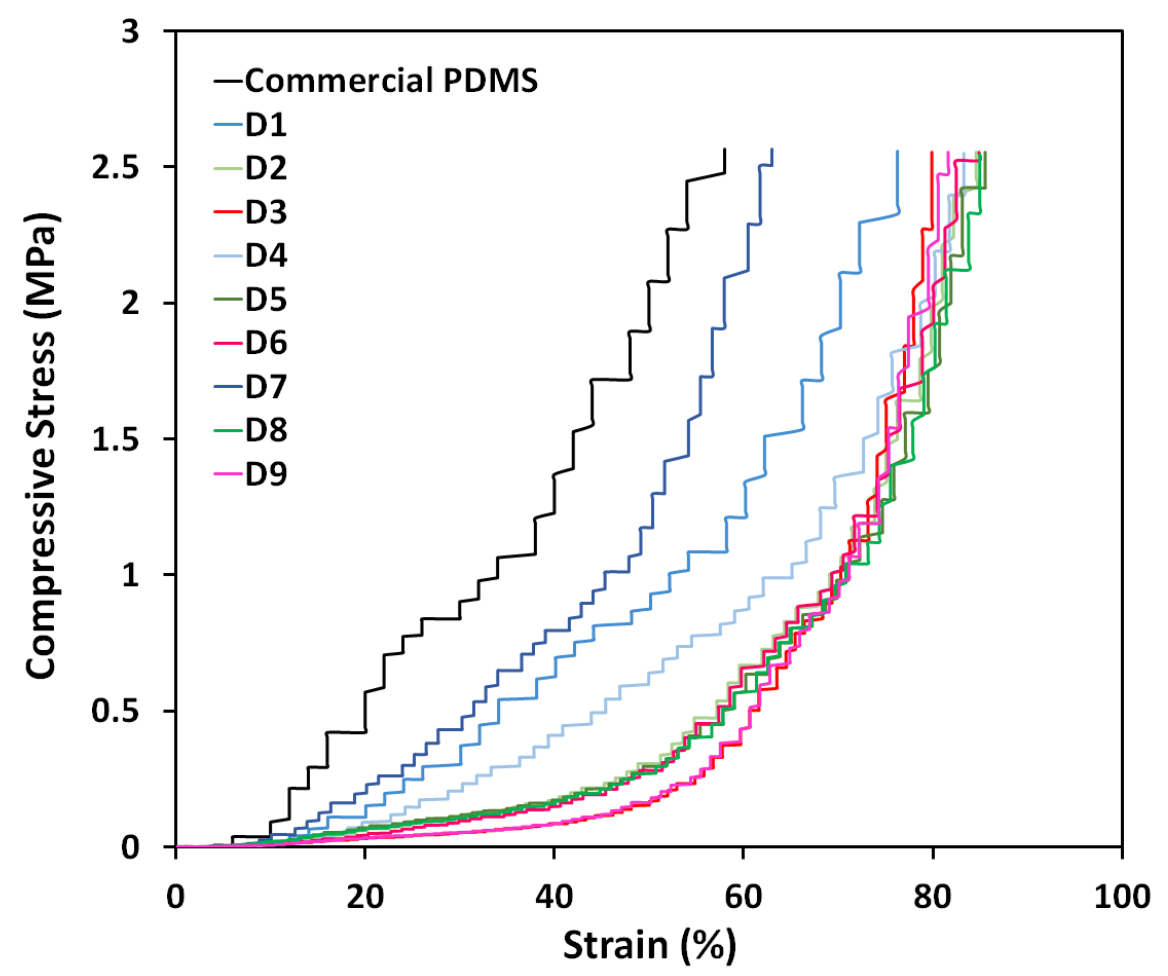

Figure S6.: Stress/strain curves showing the compression deformation of porous dialectic layers D1, D2, ...D9.

Step-Wise and repeatability/Hysteresis Response:

(a)

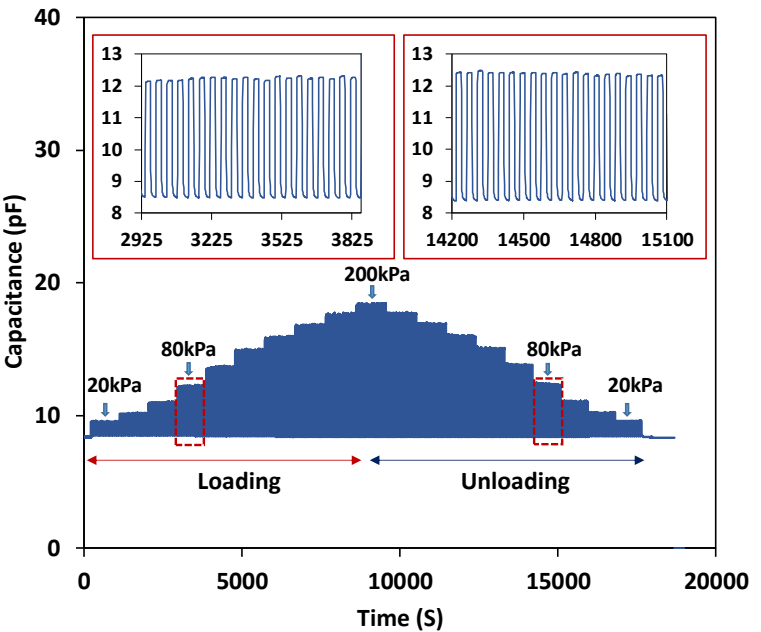

(b)

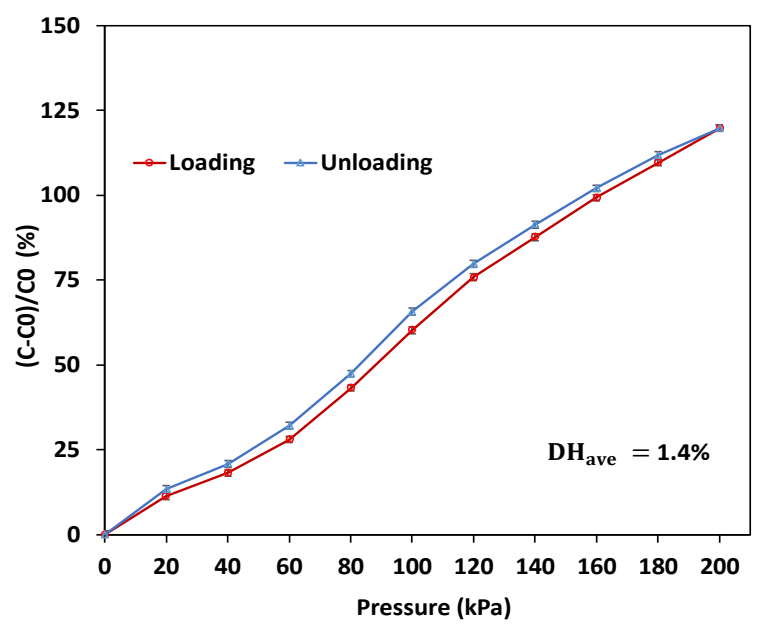

Figure S7. Repeatability of pressure sensor PS2 in pressure ranges from 0 to $200 \mathrm{kPa}$ (a) step-wise pressure response and (b) hysteresis response. 
Low Pressure Step-Wise and Repeatability/Hysteresis Response:

(a)

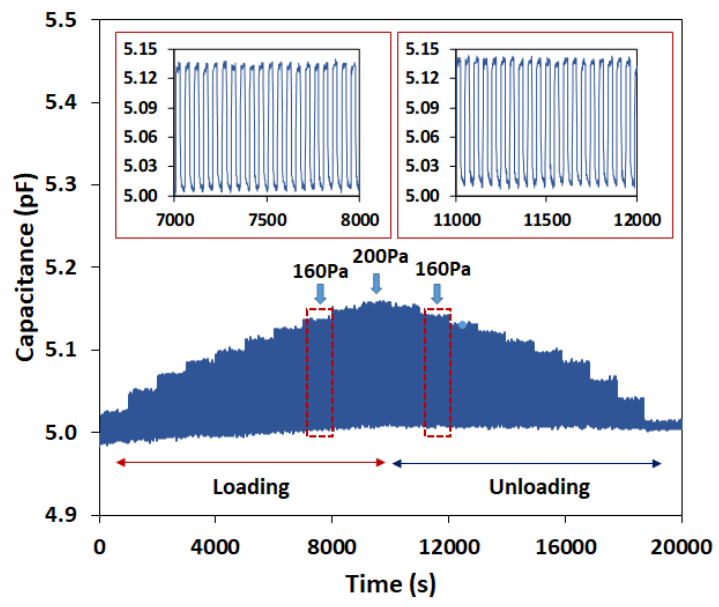

(b)

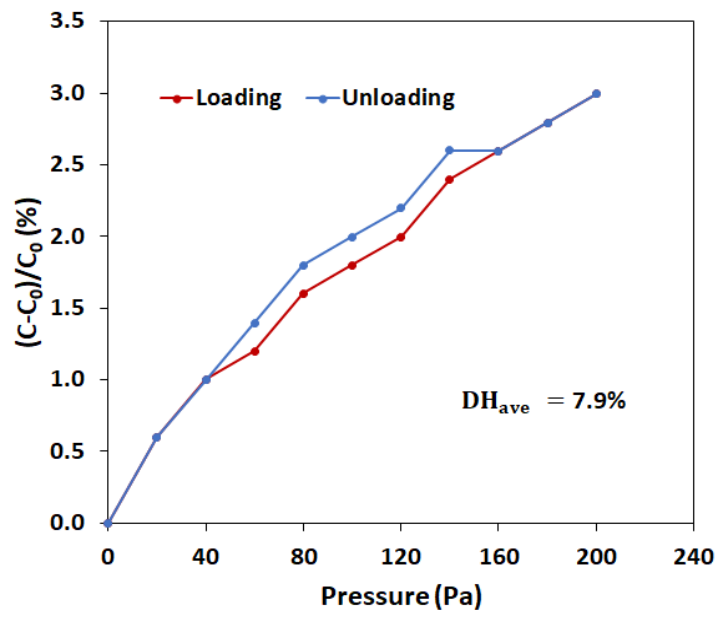

Figure S8. Repeatability of pressure sensor PS9 at low pressure ranges (<200 Pa): (a) step-wise pressure response and (b) hysteresis response.

\section{Sensor Response and Recovery Time:}

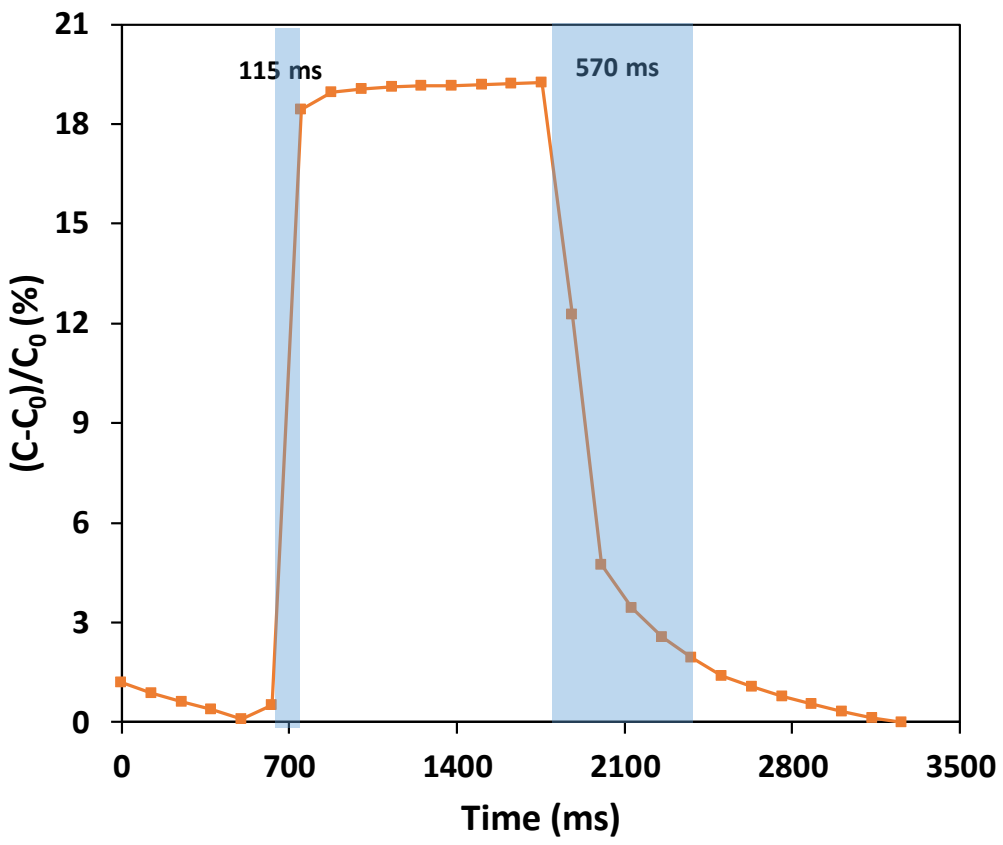

Figure S9. Response/recovery time of the pressure sensor PS2 upon loading and unloading of $50 \mathrm{kPa}$.

\section{Capacitive Response of the 16 Pressure Sensors on the Cap:}

The capacitive response of the 16 pressure sensors composed of fabric electrodes and 16 porous dielectric layers was investigated before attaching them to the cap. As a preliminary uniformity test, two fabric electrodes were attached on the stationary plate of a test stand and moving plate of a force gauge from their fabric side using double sided tape. The porous dielectric layer was then placed on the attached bottom electrode and positioned for complete overlap with the top electrode when the compression plate touches the dielectric layer (Figure S5(a)). Following this, the capacitive response of the pressure sensor was recorded for the applied pressure ranges from 2-20 kPa. Similarly, the second porous dielectric layer was placed between the top and bottom electrodes and the experiment was repeated for all the fabricated 16 dielectric layers. Figure 
S5(b) and Table S1 show the capacitive response of the 16 pressure sensors used in the fit cap for the applied pressures ranging from 2-20 $\mathrm{kPa}$.

(a)

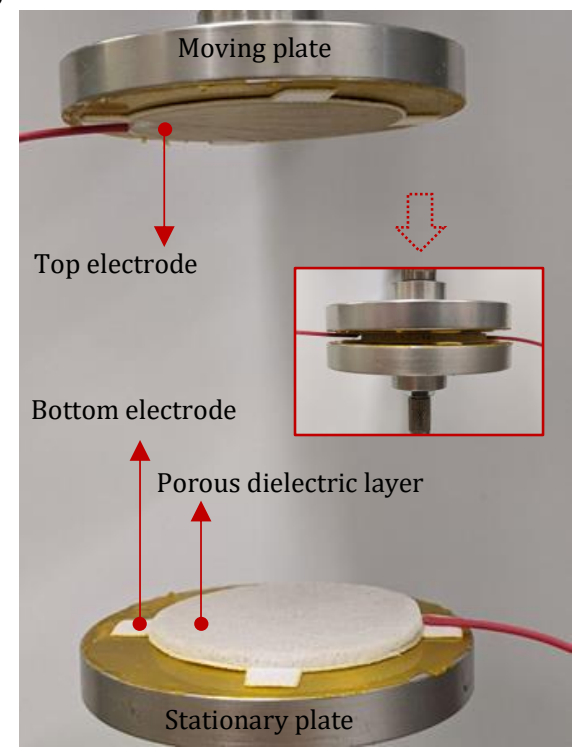

(b)

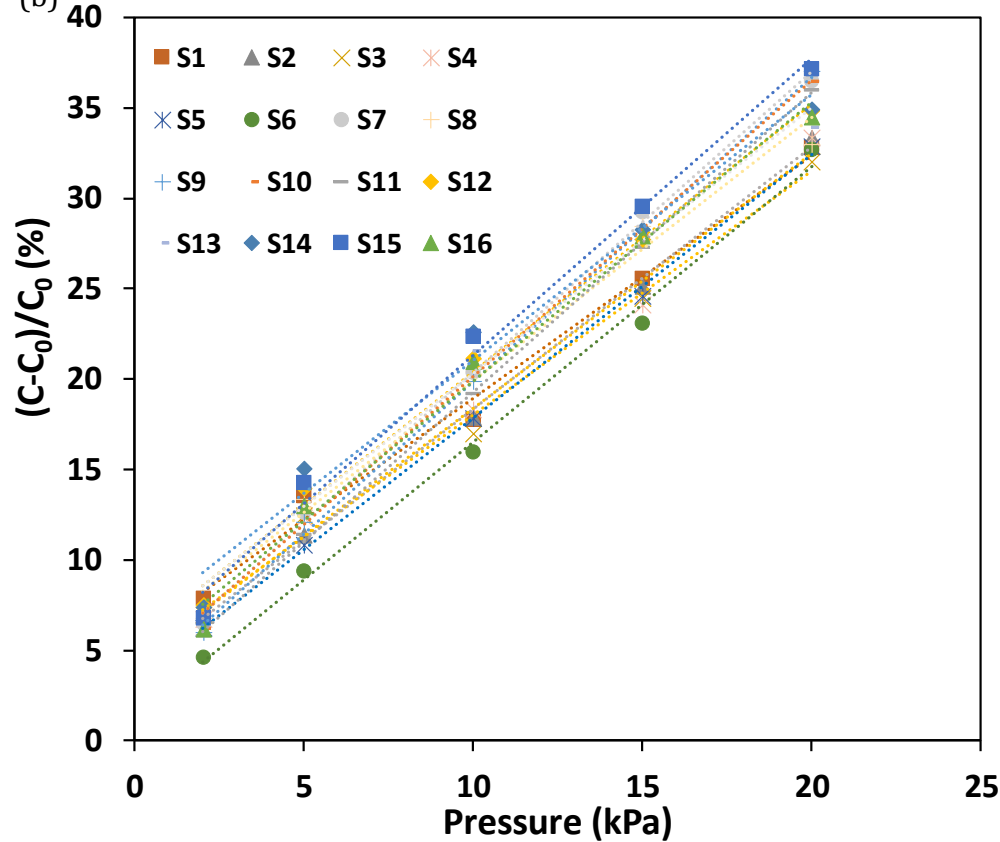

Figure S10. Preliminary investigation on the capacitive response of the pressure sensor array used in the fit cap (a) placing the pressure sensors on the force gauge and (b) relative capacitance change of the 16 pressure sensors.

Table S1. Capacitive Response of the16 Pressure Sensors Used in the Fit Cap (2-20kPa)

\begin{tabular}{lccccccc} 
Sensor & $\begin{array}{c}\text { Capacitance } \\
\text { Change }(\%)\end{array}$ & $\begin{array}{c}\text { Sensitivity } \\
{[\% / \mathrm{kPa}]}\end{array}$ & $\begin{array}{c}\text { Correction Coefficient } \\
\left(\mathrm{R}^{2}\right)\end{array}$ & Sensor & $\begin{array}{c}\text { Capacitance } \\
\text { Change }(\%)\end{array}$ & $\begin{array}{c}\text { Sensitivity } \\
{[\% / \mathrm{kPa}]}\end{array}$ & $\begin{array}{c}\text { Correction Coefficient } \\
\left(\mathrm{R}^{2}\right)\end{array}$ \\
$\mathrm{S} 1$ & 32.8 & 1.34 & 0.991 & $\mathrm{~S} 9$ & 37.0 & 1.68 & 0.998 \\
$\mathrm{~S} 2$ & 33.3 & 1.45 & 0.998 & $\mathrm{~S} 10$ & 36.5 & 1.63 & 0.996 \\
S3 & 32.0 & 1.34 & 0.992 & $\mathrm{~S} 11$ & 36.0 & 1.65 & 0.999 \\
S4 & 33.3 & 1.41 & 0.991 & $\mathrm{~S} 12$ & 34.9 & 1.47 & 0.994 \\
S5 & 32.8 & 1.45 & 0.998 & $\mathrm{~S} 13$ & 34.0 & 1.45 & 0.982 \\
S6 & 32.7 & 1.52 & 0.994 & $\mathrm{~S} 14$ & 35.5 & 1.46 & 0.982 \\
S7 & 36.5 & 1.66 & 0.998 & $\mathrm{~S} 15$ & 37.1 & 1.64 & 0.991 \\
S8 & 33.0 & 1.45 & 0.982 & $\mathrm{~S} 16$ & 34.5 & 1.54 & 0.991 \\
\hline
\end{tabular}


Table S2. Comparison of Performance Metrics of the Present Work with Recently Reported Pressure Sensors

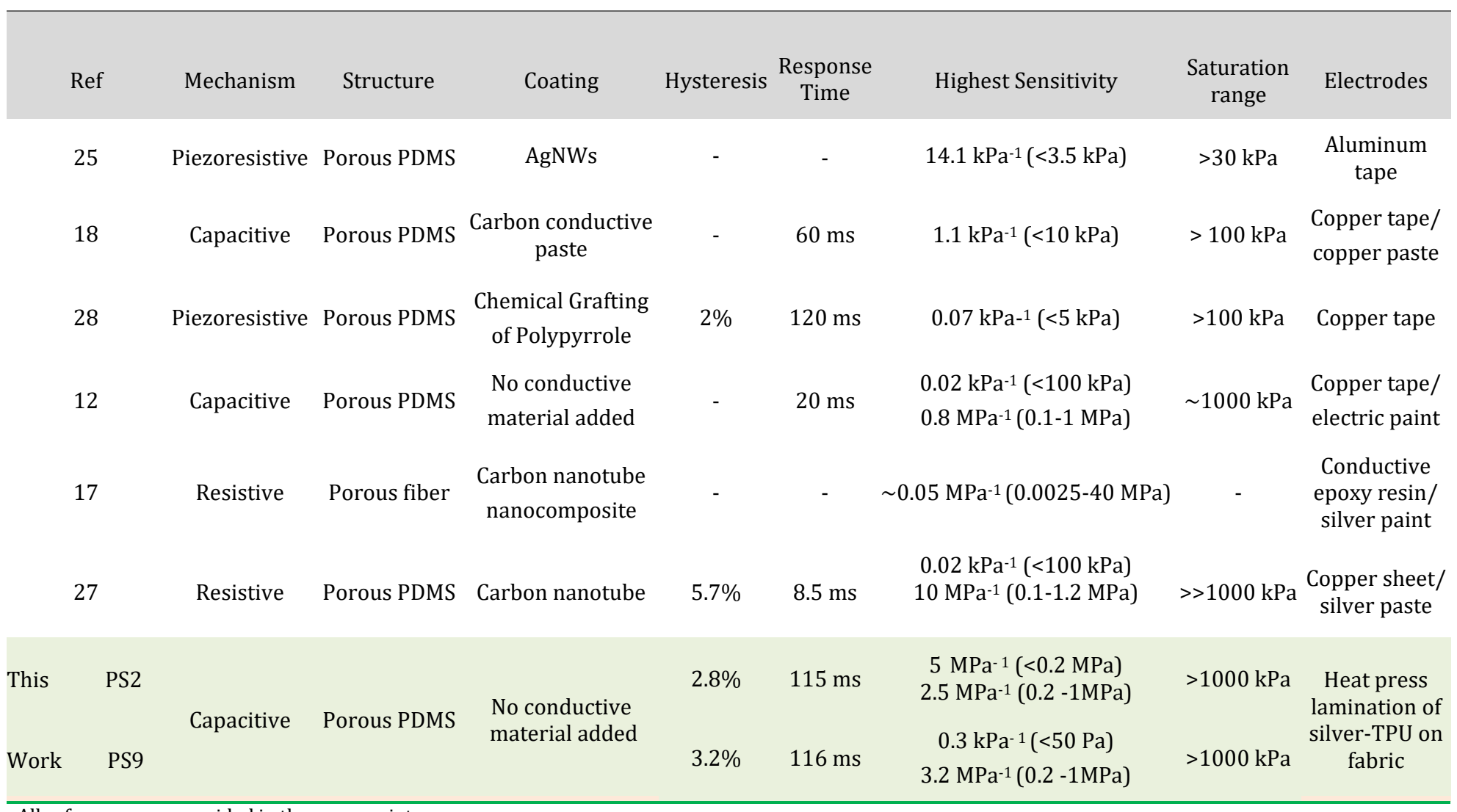

All references are provided in the manuscript. 